\title{
Engineering Oxygen Migration for Homogeneous Volume Resistive Switching in 3- Terminal Devices
}

Juan Carlos Gonzalez-Rosillo, Rafael Ortega-Hernandez, Benedikt. Arndt, Mariona Coll1, Regina Dittmann, Xavier Obradors, Anna Palau, Jordi Suñe and Teresa Puig*

Dr. Juan Carlos Gonzalez-Rosillo, Dr. Rafael Ortega-Hernandez, Dr. Mariona Coll, Prof. Xavier Obradors, Dr. Anna Palau and Prof. Teresa Puig

Institut de Ciència de Materials de Barcelona (ICMAB-CSIC), Campus de Bellaterra, 08193, Spain

E-mail: teresa.puig@icmab.es

Dr. Rafael Ortega-Hernandez and Prof. Jordi Suñe

Departament d’Enginyeria Electrònica, Universitat Autònoma de Barcelona, 08193 Bellaterra, Spain

Dr. Benedikt Arndt and Prof. Regina Dittmann

Peter Grünberg Institute, Research Center Jülich, Wilhelm-Johnen-Straße, Jülich 52425, Germany

Keywords: Resistive Switching, nanoelectronics, Oxygen Exchange, Strongly Correlated Systems

Resistive Switching effects are in a superb position to tackle the challenges for the near future of nanoelectronics and neuromorphics. Materials-wise, the outstanding properties of strongly correlated metallic perovskite oxides, in particular, those displaying Metal-Insulator Transition can be exploited for a new generation of devices based on a Volume Resistive Switching (VRS) phenomenon beyond filamentary and interface ideas. We report a full description of this new and robust physical mechanism governing VRS memory effects in mixed-valence mixed-conductor metallic La1-xSrxMnO3-y perovskites by identifying the role and rate limiting steps of oxygen exchange through oxygen partial pressure experiments. We demonstrate that oxygen migration can be smartly engineered by introducing a CeO2-x capping layer, which we further use to validate the VRS phenomenon by operating a nonvolatile and volumetric proof-of-concept gate-controlled three-terminal conductive bridge device. 


\section{Introduction}

The physical implementation of the memristor, firstly postulated by Chua ${ }^{[1]}$ and experimentally demonstrated by Strukov et al. ${ }^{[2]}$ has offered a completely new set of possibilities for memory and logic operations, such as Resistive-Random Access Memories (RRAM) and neuromorphic computing ${ }^{[3]}$. Extensive research has been carried out in last decade to implement this technology in real device architectures to overcome the scalability limits of actual electron-based technologies. ${ }^{[4]}$ The promising performance of RRAM cells in terms of endurance, retention and switching speeds justifies the large efforts performed by the community to enable their widespread use. ${ }^{[5]}$ However, there are still several fundamental and practical issues to be addressed in order to fulfill industrial requirements of reproducibility and reliability of the manufactured devices. That is the case of actual devices relying on widely used filamentary-type resistive switching (RS) mechanism, ${ }^{[6]}$ where the main difficulty lies on the random formation (and subsequent rupture) of the aforementioned filament (which determines whether the device is in an ON or an OFF state, respectively), or leakage currents that could arise in the devices, as well as other issues intrinsically arising from the high non-linearity electric field dynamics of the materials. ${ }^{[7]}$ Additionally, there is the need of a full comprehension of the underlying physical mechanism in some of the active RS materials, ${ }^{[6,7]}$ to provide the final boost from the material's perspective to engineering of the devices.

From the materials point of view, simple binary oxides are widely studied and are viable materials for its simplicity, CMOS compatibility and promising performance, and thorough research is being conducted to tackle the issues exposed above. Alternatively, the interest of strongly correlated systems with functional properties, such as Metal-Insulator Transition (MIT), is growing ${ }^{[8-10]}$ and heading towards applications. The exotic intrinsic properties of these complex oxides could lead to novel design of devices (for a complete review, see ${ }^{[8]}$ ) with enhanced performance and functionalities, specially through the control of oxygen 
kinetics, which has dramatic implications on the functional properties ${ }^{[11]}$. Previous studies have related the resistive switching effect in metallic perovskite oxides to their intrinsic MIT properties even through film thickness up to $10 \mathrm{~nm}$ suggesting a Volume Resistive Switching mechanism. ${ }^{[10,12,13,36]}$ Oxygen release from the crystal lattice would generate oxygen vacancies in the system, concomitant with a decrease of the charge carriers in the system and a valence state reduction $\left(\mathrm{Mn}^{+4} \rightarrow \mathrm{Mn}^{3+}\right)$. The advantages of showing bulk RS characteristics in these metallic perovskite oxides, instead of usual filament or filamentary ones in dielectric oxides, are i) more robust switching performance, ii) highly spatial control of the switching event, iii) easier circuit integration due to the initial metallic character of the films and iv) high reproducibility of the effect, since it is based on an intrinsic property of the material itself. This manuscript demonstrates the power of controlling the oxygen exchange mechanism in the Volume Resistive Switching phenomenon by understanding and engineering of the RS effect in a metallic complex oxide displaying MIT, whose functionalities are exemplified with a proof-of-concept three-terminal (3-T) device. In particular, we have chosen $\mathrm{La}_{0.8} \mathrm{Sr}_{0.2} \mathrm{MnO}_{3}$ (LSMO) as the mixed-valence-mixed conductor metallic oxide and $\mathrm{CeO}_{2-\mathrm{x}}$ as the oxygen reservoir buffer layer. ${ }^{[13]}$ We provide an explanation supported on strong evidences for an oxygen migration-mediated RS effect in LSMO films (and extensible to other metallic complex oxides, such as cuprates and nickelates ${ }^{[10]}$ ), while material engineering through the use of a bilayer system with an oxygen buffer layer offers novel functional devices as the proof-of-concept 3-T device presented. This device shows the implementation of a proof-ofconcept gate-controlled volume resistive switching system where the current conduction along the $\mathrm{CeO}_{2-\mathrm{x}} / \mathrm{LSMO}$ bilayer bridge is modulated by inducing resistive switching in a nonvolatile, tunable manner, for the first time, up to $40 \mathrm{~nm}$ in material thickness. The huge potential of Mott transitions for envisioning new devices, with particular focus on 3-T devices, has attracted some recent interest (including ionic liquid gating). ${ }^{[9,14-16]}$ Even more, metallic perovskite oxides displaying MIT have been shown to change their physical properties in a 
homogenous manner through their volume upon generation of oxygen vacancies, ${ }^{[10,12,17]}$ although major effects might occur at the interface, including a probable phase transition. ${ }^{[18]}$ The proof-of-concept that we report here provides a device realization after understanding and controlling the volume switching process on a mixed-valence-mixed conductor model system as $\mathrm{La}_{0.8} \mathrm{Sr}_{0.2} \mathrm{MnO}_{3}$ in which the switched thickness of up to $40 \mathrm{~nm}$ is demonstrated. This emerging homogeneous volume resistive switching may launch new applications in neuromorphic computing, ${ }^{[3,19]}$ where specially Mott systems are attracting attention from the research community, ${ }^{[3,20-22]}$ but also in the RRAM field due to the robustness of the effect. $^{[10,13]}$

\section{Atmosphere Dependent I-V Curves}

The RS characteristics of bare epitaxial LSMO thin films (Figure. 1a) were first investigated in air (ambient conditions) and in vacuum $\left(\mathrm{P}=10^{-5}\right.$ mbar) conditions (Figure $\left.1 \mathrm{~b}\right)$ with the microprobes in direct contact with the LSMO layer (the voltage cycle direction is indicated by arrows). A transition from the Low Resistance State (LRS or ON state) to the High Resistance State (HRS or OFF state) is induced at negative bias voltage. For both atmospheres, the generated HRS is non-volatile, and it switches back to the LRS at a certain positive bias, $\mathrm{V}_{\mathrm{HR} \rightarrow \mathrm{LR}}$. Repeatability was assessed by performing $100 \mathrm{I}-\mathrm{V}$ curves at the same location. Reproducible and stable RS characteristics are obtained in both atmospheres. However, the generated HRS at ambient conditions presents 1000 times higher resistance than that generated in vacuum conditions. The resistance value of the LRS and the HRS were evaluated at $\mathrm{V}_{\text {bias }}=0.5 \mathrm{~V}$ and the cumulative probability was calculated and plotted in Figure $1 \mathrm{~d}$. By comparing the resistance of the LRS we found lower values at vacuum conditions (LRS

resistance in ambient conditions $\boldsymbol{R}_{L R S}^{a m b} \sim 10^{5} \Omega$, and HRS resistance in vacuum, $\boldsymbol{R}_{L R S}^{v a c} \sim \mathbf{1 0}^{4} \Omega$ ), that may be attributed to a better mechanical contact between the tip and the sample under vacuum conditions than in air. It is worth to note that the main impact by changing the base 
pressure is obtained in the HRS. While in ambient conditions a large resistance ratio from HRS to LRS of $\mathrm{r}_{\mathrm{amb}}=\mathrm{R}_{\mathrm{HRS}} / \mathrm{R}_{\mathrm{LRS}} \approx 10^{3}-10^{4}$ is found, the ratio observed in vacuum, $\mathrm{r}_{\mathrm{vac}}$, is extremely diminished down to a value of $r_{\mathrm{vac}} \approx 2$. Therefore, we demonstrate that the RS is produced through an atmosphere exchange with ambient which has strong impact on the generated HRS. For a deeper understanding of the phenomenon, I-V curves were also acquired at different oxygen partial pressures (Figure 1c). In particular, three different oxygen partial pressures, $\mathrm{P}\left(\mathrm{O}_{2}\right)$, were used, namely $0.74,0.22$ and 0.019 mbar. To assure the proper stabilization of the oxygen atmosphere, measurements were performed at least one hour after the device showed the desired pressure set point. The same sweeping protocol as in the previous measurements was applied. Reproducible and stable sweeps are observed in the tested range of pressure. The resistance values at $\mathrm{V}_{\text {bias }}=0.5 \mathrm{~V}$ for the HRS and LRS branches are extracted and their cumulative probability plots are shown in Fig. 1e. The LRS value at every $\mathrm{P}\left(\mathrm{O}_{2}\right)$ tested presents roughly the same value, $\mathrm{R}_{\mathrm{LRS}}=4 \cdot 10^{4} \Omega$ showing no dependence on the external pressure. Interestingly, the generated HRS presents a smooth increase of the resistance value upon increasing the external $\mathrm{P}\left(\mathrm{O}_{2}\right)$. No significant differences were observed in the voltages at which the resistive transitions occur. These measurements highlight the key role that oxygen motion plays in the resistive switching behavior of metallic perovskite oxides. We now evaluate the switching performance in $\mathrm{Ag} / \mathrm{CeO}_{2} / \mathrm{LSMO}$ cells (Figure 1 (f,g) in air and vacuum conditions. In this case, the general shape of the I-V curves is very similar in both atmospheres. Highly reproducible voltage sweeps were obtained after an initialization step (see Supporting Information, Figure S2). However, some changes are observed between them. The resistance value of the LRS and the HRS were evaluated at $\mathrm{V}_{\text {bias }}=0.2 \mathrm{~V}$ and the cumulative probability was calculated and plotted in Fig. 1h. Compared to the response in air, an overall increase of the resistance at vacuum conditions in both the LRS and the HRS is found. This increase in the resistance might be attributed to the absence of hydroxyl ions at the topmost layer of the $\mathrm{CeO}_{2-x}$ layer, ${ }^{[23,24]}$ that may affect the readout of both resistive states. 
This modification of the $\mathrm{Ag} / \mathrm{CeO}_{2-\mathrm{x}}$ interface could act as a voltage divider and explain as well the higher voltage requirements in ambient than in air. Remarkably, neither the latter fact nor the external atmosphere produces a deep change in the overall performance of the device, since both the I-V shapes and a very similar resistance ratio of $\mathrm{R}_{\text {off }} / \mathrm{R}_{\mathrm{on}} \sim 10^{4}$ are found at low pressures and ambient conditions. This experiment undoubtedly shows that anion exchange occurs between the $\mathrm{CeO}_{2-\mathrm{x}}$ and the LSMO layer and not through the interface with the environment or with the metal electrode. ${ }^{[25,26]}$ In also, variations on the atmospheric conditions have been shown to potentially affect the device performance. ${ }^{[23]}$ The robustness of the effect is therefore proven. It remains open for further understanding the interesting lower operational voltages in the $\mathrm{Ag} / \mathrm{CeO}_{2-\mathrm{x}} / \mathrm{LSMO}$ heterostructures and sharper transitions than in the bare LSMO case. For the former, it is very likely that oxygen exchange reactions with the atmosphere require higher voltages than simple anion exchange between the two oxide layers. For the latter, we believe that the suppression of the atmosphere exchange in the bilayer case is responsible of a sharp transition, as one would expect from a direct exchange between the oxides (where release and incorporation from the ambient do not occur). Preliminary pulsing data (Supplementary Information) reveals switching times as low as 100 ns at voltages, which leads to a very low power consumption for each writing/erasing cycle. Similar switching speeds have been explained in other oxides as a combination of field-driven and Joule-heating self-accelerated processes. ${ }^{[18,26,27]}$ Systematic pulsing studies and adequate modelling needs to be carried out to understand the balance of both contributions in determining the switching kinetics.

\section{Volume Resistive Switching Mechanism}

Despite being one of the "simplest" examples of solid-gas chemical reaction, oxygen exchange from a film to the exterior, is a very intricate process that is summarized in four steps: ${ }^{[28-31]}$ Adsorption (from $\mathrm{O}_{2}$ gas to the surface of the solid), dissociation $\left(\mathrm{O}_{2}\right.$ molecule is split at the surface), surface migration $\left(\mathrm{O}^{-}\right.$or $\mathrm{O}^{2-}$ ions diffuse at the surface to find oxygen 
vacancies) and finally, incorporation / release $\left(\mathrm{O}^{2-}\right.$ ions incorporate /release into/from the lattice by recombining with/creating an oxygen vacancy). In the particular case of LSMO, surface migration is considered the rate determining step (RDS) of the reaction. ${ }^{[30]}$ On the other hand, in our RS experiments, the release of oxygen from the lattice occurs due to an electrochemical reaction between the adsorbate species and an anion at the crystal interface, thus creating an oxygen vacancy in the lattice and forming an $\mathrm{O}_{2}$ molecule at the surface. In our case, the three first steps are common for both the oxygen release and incorporation processes and are crucial to understand the impact of varying the oxygen partial pressure, $\mathrm{P}\left(\mathrm{O}_{2}\right)$ in the switching performance. In the adsorption step, physisorption and chemisorption processes of $\mathrm{O}_{2}$ molecules should be considered. The energy of the former adsorbate is very small $\left(E_{a d s}=-0.25 e V\right)$ whereas the latter $\left(\mathrm{O}^{2-}\right)$ is more likely to be formed $\left(E_{a d s}=-2 e V\right)$ through a charge transfer process. ${ }^{[32]}$ Oxygen vacancies and other defects might act as preferential adsorption sites. However, the concentration of them is rather low and adsorption processes occur in clean surfaces as well. Then, the $\mathrm{O}^{2-}$ adsorbates are dissociated into two $\mathrm{O}^{-}$ads. After the dissociation, $\mathrm{O}^{-}$species need to diffuse to find an oxygen vacancy in the system to then recombine. This surface migration step is considered the rate determining step of the whole oxygen surface exchange reaction, especially at room temperature. The formation of charged adsorbates $\left(\mathrm{O}^{2-}, \mathrm{O}_{2}{ }^{2-}, \mathrm{O}^{-}\right)$and their mutual electrostatic interaction lead to a certain coverage degree for each species ${ }^{29}$, that can be described in first approximation as

$\theta_{o_{n}^{j}}=K_{a d s} \cdot\left(p\left(O_{2}\right)^{\frac{n}{2}} \cdot \exp \left(-\frac{j e_{0} \chi_{\max }}{k T}\right)\right.$

where $\mathrm{j}$ is the adsorbate charge, $\mathrm{n}$ the number of oxygen atoms it contains and $\mathrm{K}_{\mathrm{ads}}$ the mass action constant of adsorption. $\chi_{\max }$ is the surface potential drop built up in case of full coverage. Therefore, the surface coverage can change orders of magnitude upon changes in the oxygen partial pressure, $\mathrm{p}\left(\mathrm{O}_{2}\right)$, thus playing a key role to understand the resistive switching properties in LSMO thin films. However, the exact calculation of the coverage is 
not trivial, since different crystalline surfaces usually lead to modified coverage degree, and also it is influenced by steps and grain boundaries densities. Calculations show that the atomic adsorbates $\left(\mathrm{O}^{-}\right)$dominates the coverage ${ }^{33}$ with a larger presence than the molecular adsorbates $\left(\mathrm{O}^{-2}, \mathrm{O}^{2-}\right)$. In transition metal oxides it is estimated that $10^{-2}-10^{-3}$ adsorbates per unit cell should be achieved at a $\mathrm{P}\left(\mathrm{O}_{2}\right)=1$ bar. $^{[30]}$ The three steps, shown in Figure 2a, draw a framework in which adsorbates, mainly $\mathrm{O}^{-}$species, are adsorbed at the surface with a certain coverage degree which has a strong dependence on the $\mathrm{p}\left(\mathrm{O}_{2}\right)$.

We now discuss the local oxygen exchange induced in full reversible resistive switching event. It should be noted that $\mathrm{I}-\mathrm{V}$ measurements are not performed in the usual top-bottom configuration. Instead, a top-top configuration is used as was detailed in our previous work. ${ }^{[10]}$ In essence, the dynamic simulation of the system indicated that, when the film is under negative bias and the tip grounded, the switching front is initially induced close to the interface and it is continuously pushed downwards as the local resistivity is changed dynamically during the switching event.

Therefore, under negative bias, $\mathrm{O}^{2-}$ species in the crystal structure are attracted to the interface with the grounded tip. In addition, the electric field induces the following electrochemical reaction (Figure $2 b)$ :

$O_{x}^{o} \rightarrow \frac{1}{2} O_{2}+V_{o}^{-}+2 e^{\prime}$

When the generated $\mathrm{O}^{2-}$ ads (or $\mathrm{O}_{\text {ads }}^{-}$) is at the surface, it needs to diffuse to meet another $\mathrm{O}^{-}{ }_{\text {ads }}$ to form an $\mathrm{O}_{2}$ molecule. As explained before, this is the RDS of the whole oxygen exchange reaction. When the RS is produced under low $\mathrm{p}\left(\mathrm{O}_{2}\right)$, adsorption coverage $\Theta$ is low and so the formation of $\mathrm{O}_{2}$ molecules will not be favored because the surface diffusion length is much larger. As a consequence, the overall number of exchanged oxygen ions through the surface will be strongly reduced, and therefore, the $\mathrm{R}_{\mathrm{off}} / \mathrm{R}_{\mathrm{on}}$ ratio is heavily decreased. For this reason, at low $\mathrm{p}\left(\mathrm{O}_{2}\right)$ only a small variation in the charge carriers can be produced and therefore a very 
small resistance ratio $(\sim 2)$ is induced. Once the HRS has been induced in the region below the tip, a high enough electric field at positive bias generates the reverse electrochemical reaction (Figure 2c):

$\div O_{2}+V_{o}^{w}+2 e^{y} \rightarrow O_{x}^{O}$

Oxygen adsorbates at the surface are incorporated into the system via this electrochemical process and therefore, the oxygen stoichiometry is restored. Note that this has an associated valence change in the Mn metal cations, which simultaneously increases the number of carriers leading the system into a more metallic conduction state.

This complex electrochemical reaction is avoided when using the $\mathrm{CeO}_{2-\mathrm{x}}$ oxygen reservoir capping layer through a direct oxygen exchange between the LSMO and the capping layer. We had already demonstrated that $\mathrm{CeO}_{2-\mathrm{x}}$ films deposited by Atomic Layer Deposition on $\mathrm{Pt}$ substrates did not show any RS characteristic on their own under the employed conditions in this work $^{13}$. Let us consider a device as that shown in Fig. 1f, that after the initialization step and some stabilizing I-V cycles, it is found to be in its HRS in the $\mathrm{La}_{0.8} \mathrm{Sr}_{0.2} \mathrm{MnO}_{3}$ region under probe A (Figure 2d). When positive voltage is applied on probe $\mathrm{B}$, the negatively charged oxygen ions at the $\mathrm{CeO}_{2-\mathrm{x}}$ layer are repulsed towards the $\mathrm{CeO}_{2-\mathrm{x}} / \mathrm{La}_{0.8} \mathrm{Sr}_{0.2} \mathrm{MnO}_{3}$ interface beneath the electrode A by the effect of the voltage bias polarization, where they are exchanged and recombined with the oxygen vacancies within the $\mathrm{La}_{0.8} \mathrm{Sr}_{0.2} \mathrm{MnO}_{3}$ layer producing a HRS $\rightarrow$ LRS transition driven by the $\operatorname{MIT}^{[10,13]}$ (Figure 2d). At the following negative voltage applied on probe $\mathrm{B}$ in this new state, the reverse voltage bias polarization produces that the negatively charged oxygen ions can be released from the LSMO layer and exchanged with the $\mathrm{CeO}_{2-x}$ layer leading to a LRS $\rightarrow \mathrm{HRS}$ (Figure 1g) transition in the same region below the electrode A (Figure 2e).

First works describing the RS effect in lanthanum strontium manganites already remarked the extended nature of the effect ${ }^{[37]}$, even in reports far away from the resistive switching 
community $^{[17]}$. It was later suggested the impact that an exchange with the environment might have on the switching ${ }^{[12]}$, specially linked to the MIT of these systems ${ }^{[10]}$. In this sense, this is the first time that a direct evidence of the impact of the atmosphere has on the resistive switching properties of manganites. Our findings and modelling are compatible with recent in-situ TEM, observations by in-situ TEM microscopy experiments, where an oxygen vacancy-driven structural and resistive phase transitions were found ${ }^{[18]}$. These previous reports pointed towards a bulk limited conduction mechanism in LSMO films rather than a Schottky barrier mechanism. To confirm that, we further analyzed the conduction mechanism through the tip-oxide interface in both resistive states by analyzing the $d(\operatorname{Ln}(I)) / d(\operatorname{Ln}(V))$ vs $\mathrm{V}^{0.5}$ (see Supporting Information, Fig. S6 and Refs. 36 and 42.). The derivative term shows a constant value equal to 2.5 for the LRS and 3 for the HRS, which corresponds to a complex behavior close to a bulk-limited process through a Space Charge Layer Conduction mechanism $(d(\operatorname{Ln}(I)) / d(\operatorname{Ln}(V))=2)$. However, we emphasize that the complex behavior of the I-V curves cannot be attributed to a single electrode or bulk conduction mechanism, as shown with this analysis. The oxygen exchange mechanism univocally implies a valence change in the $\mathrm{Mn}$ ions due to the coordination of the cation with an oxygen octahedron and a concomitant modification of the transport properties ${ }^{43-47}$. Through these experiments we have been unable to estimate the magnitude of the valence change, which requires further spectroscopic techniques to elucidate the magnitude of the change. In the bilayer case, by introducing the $\mathrm{CeO}_{2}$ capping layer we are able to reduce the switching voltages when compared to the single manganite case as a consequence of avoiding oxygen exchange with the atmosphere. Further studies are required to fully understand the implications of the $\mathrm{CeO}_{2-\mathrm{x}}$ capping layer and the top electrode on the overall performance of the devices. Of special interest is the understanding of the transport mechanism at the $\mathrm{CeO}_{2-\mathrm{x}} / \mathrm{LSMO}$ interface, which remains elusive at this stage. However, we discard that the switching only arises from the $\mathrm{Ag} / \mathrm{CeO}_{2}$ capping layer as demonstrated in Ref. 13. 


\section{Three-Terminal Device}

We are now ready to present the implementation of a proof-of-concept gate-controlled volume resistive switching system where the current conduction along a $\mathrm{CeO}_{2-\mathrm{x}} / \mathrm{LSMO}$ bilayer bridge is modulated by inducing a volumetric resistive switching up to $40 \mathrm{~nm}$ through thickness in a non-volatile, reversible and tunable manner, for the first time.

The fabricated device consists of a $140 \mu \mathrm{m}$ x $30 \mu \mathrm{m} \mathrm{CeO}_{2-\mathrm{x}} / \mathrm{LSMO}$ track with $50 \mathrm{~nm} \mathrm{Ag}$ electrodes sputtered on it, as shown in Figure 3a. Electrodes A and B serve to measure the resistance along the bridge at low voltages, while $C$ and Gate $(G)$ electrodes are used for writing/erasing the $\mathrm{CeO}_{2-\mathrm{x}} / \mathrm{LSMO}$ track under the gate electrode area. Positive voltage was applied to the C electrode while the G electrode was electrically grounded (same electrical configuration as in the previous experiments). An initialization step was performed to set the device to the desired initial state (Figure S3).

The evaluation of the volume resistive switching in fresh three-terminal devices is defined in the following protocol: A first low voltage sweep $( \pm 1 \mathrm{~V})$ is applied along the track between the A-B electrodes in order to determine the initial state of the device without disturbing it. In the present case (see Figure 3e), the resistance value of $1 \mathrm{M} \Omega$ was measured, which confirms that any breakdown process occurred neither in the $\mathrm{CeO}_{2-\mathrm{x}}$ nor in the $\mathrm{La}_{0.8} \mathrm{Sr}_{0.2} \mathrm{MnO}_{3}$ layer at this stage, as no hysteresis is observed under the applied sweep. As a second stage of the measurement protocol, a positive voltage up to $+3 \mathrm{~V}$ is applied between the gate and the $\mathrm{C}$ electrode producing a transition of the resistance stage. In the present case (Figure 3d), a transition from HRS to LRS in the LSMO layer under the gate electrode G is attained. Subsequently, a low voltage reading measurement (A-B electrodes) is performed on the track where it is observed that the resistance has decreased down to a value of $66 \mathrm{k} \Omega$ denoted in Figure 3e as the "LRS" curve. Finally, a HRS state in the LSMO layer under the gate electrode is written by applying a negative voltage on the electrode $\mathrm{C}$ up to $-3 \mathrm{~V}$. This is 
confirmed by evaluating the track resistance again, and demonstrating that the initial value of $1 \mathrm{M} \Omega$ is recovered (Figure 3e - "HRS" curve). These results demonstrate that volume resistive switching is able to modulate the conduction characteristics along the bilayer track by modifying the resistance of the area under the gate electrode in a non-volatile manner. Figure 3f shows the resistance state measured on the track after each writing/erasing operation over 20 cycles. The resistance ratio of $\sim 15$ is maintained indicating good repeatability of the resistive switching phenomena used to modulate the conduction along the track. Notice that this value depends strongly on the geometry employed, which was not optimized to maximize the resistance ratio readout, and could be largely improved by modifying the geometries of the device. In fact, larger resistances ratios are expected if a 4-point measurement was employed or larger volume was designed to be the switching element under the gate.

A parallel resistance model based on two resistance states (HRS and LRS) has been used to determine the modified thickness of the $\mathrm{La}_{0.8} \mathrm{Sr}_{0.2} \mathrm{MnO}_{3}$ layer (see Figure 3c and Supporting information). Solving the equations for the equivalent resistance circuit and relating the measured parameters of resistance ratio $\left(\mathrm{R}_{\mathrm{HRS}} / \mathrm{R}_{\mathrm{LRS}}\right)$ of $10^{3}$ and $\mathrm{La}_{0.8} \mathrm{Sr}_{0.2} \mathrm{MnO}_{3}$ resistivity of $4.8 \mathrm{~m} \Omega \cdot \mathrm{cm}$ in LRS with the geometry of the three-terminal structure, a value of $38.8 \mathrm{~nm}$ has been obtained, close to the $40 \mathrm{~nm}$ thickness of the $\mathrm{La}_{0.8} \mathrm{Sr}_{0.2} \mathrm{MnO}_{3}$ layer, which indicates that the whole thickness of the $\mathrm{La}_{0.8} \mathrm{Sr}_{0.2} \mathrm{MnO}_{3}$ layer has been electrically switched between the two different resistance states. This confirms that the volume resistive switching is homogeneously distributed across the whole $\mathrm{La}_{0.8} \mathrm{Sr}_{0.2} \mathrm{MnO}_{3}$ area under the gate electrode and it is not produced by formation/rupture of local filaments inside of the switching layer. The $\mathrm{CeO}_{2}$ resistance has not been considered in the model since it presents a fixed value that would be added at both HRS and LRS on the reading operation through the A-B contacts. Further evidence that switching performance has spread through the LSMO bulk comes from the fact that the modified thickness $(\sim 40 \mathrm{~nm})$ is much larger than the CeO2 thickness $(10 \mathrm{~nm})$. 
Three-terminal devices based on correlated oxides for the use of Field-Effect Transistors $(\mathrm{FET})^{[8,14]}$ have been largely studied showing large potential for applications. Of special interest is the exceptional performance found in 3-T devices based on Li-ion migration ${ }^{[38]}$. However, there is still a lack of three-terminal devices based purely on memristive responses that could fully exploit the non-volatility and fast switching times of memristors. Remarkable attempts were done in 3-Terminals $\mathrm{TiO}_{2}{ }^{[39]}, \mathrm{ZnO}^{[40]}$ and $\mathrm{SrTiO}_{3}{ }^{[41]}$, where all the authors agreed on the large potential of the 3-T architecture to continuously tune the resistive state and achieve performances and integration densities able to emulate neuronal and synaptic process through neuromorphic chips. The $\mathrm{CeO}_{2-\mathrm{x}} / \mathrm{La}_{0.8} \mathrm{Sr}_{0.2} \mathrm{MnO}_{3}$ 3-Terminal architecture proposed here presents large potential to tune the resistive state in a non-volatile robust manner in the LSMO films with tunable resistive states and promising switching speeds $(\sim 100$ ns, see Supporting Information), faster than any 3-Terminal architecture proposed to date, which leads to a rough estimation of $\sim 50 \mathrm{pJ} /$ write operation. Further characterization to explore the limits of the neuromorphic computing capabilities of these systems is still required.

\section{Conclusion}

We provide an oxygen exchange model to understand the Volume Resistive Switching effect of metallic $\mathrm{La}_{1-\mathrm{x}} \mathrm{Sr}_{\mathrm{x}} \mathrm{MnO}_{3-\mathrm{y}}$ (LSMO) films exhibiting a Metal Insulating Transition (MIT) by means of atmosphere dependent measurements. The surface coverage of oxygen adsorbates, which is pressure-dependent, determines the anions probability exchanged at the surface. We present a smart and robust way to control the oxygen exchange requirements for this phenomenon by introducing an ionic conducting layer such as $\mathrm{CeO}_{2-x}$. In such a heterostructure, the anionic exchange is not regulated by the environment, but through a direct exchange between the LSMO and the $\mathrm{CeO}_{2-x}$ layers. By employing the MIT properties of the LSMO films to generate Volume RS characteristics, we have introduced a proof-of-concept 3$\mathrm{T}$ device, in which the lateral conduction of the bridge is controlled by the resistive state (LRS 
or HRS) in the gate electrode. We have shown that even the full thickness of the film (40 nm) could be electrically switched. This provides new insights into device fabrication and control of the switching elements in a three-terminal configuration fully exploiting the strongly correlation of manganite and similar perovskite systems.

\section{Experimental Section}

Fabrication methods: Chemical solution deposition (CSD) of $40 \mathrm{~nm} \mathrm{La}{ }_{0.8} \mathrm{Sr}_{0.2} \mathrm{MnO}_{3}$ (LSMO) thin films were prepared from stoichiometric amounts of lanthanum, strontium and manganese acetates dissolved in acetic acid with a molar concentration $0.3 \mathrm{M}$ with respect to the $\mathrm{Mn}$, stirred at $50^{\circ} \mathrm{C}$ in a hot plate, for $10 \mathrm{~min}$. All chemicals are of analytical grade and used as received without further purification. The LSMO precursor solution was spin coated on $5 \mathrm{~mm}$ x $5 \mathrm{~mm}(100)$ insulating $\mathrm{SrTiO}_{3}$ single crystal substrates and heated at $3^{\circ} \mathrm{C} / \mathrm{min}$ in a tubular furnace to $900-1000^{\circ} \mathrm{C}$ for $30 \mathrm{~min}$ in oxygen $(0.6 \mathrm{l} / \mathrm{min})$. For the bilayer system, 10 nm CeO2 films were deposited by atomic layer deposition (ALD) on LSMO/STO samples at $275^{\circ} \mathrm{C}$ using alternate pulses of tetrakis(2,2,6,6-tetramethyl-3,5-heptanedionato) cerium (IV), Ce(thd)4, and ozone. Note that pseudo-boost conditions were used to ensure the evaporation and delivery of the Ce(thd)4 precursor to the reaction chamber. High purity N2 (99.9995\%) was used as carrier and purge gas with a gas flow of 20-50 sccm. The properties of the LSMO are fully preserved after the $\mathrm{CeO} 2$ deposition as published elsewhere. ${ }^{[34,35]}$ For the fabrication of the 3-T devices, a standard photolithography process was implemented to define metallic contacts by sputtering of Ag. The thickness of these Ag contacts was 50nm. A second photolithography step was performed in order to define the structures which have been patterned through ion etching process performed under Ar plasma.

Electrical characterization: The atmosphere-dependent measurements were performed in a home-made microprobe station equipped with a Keithley 2611A source meter operated with 
customized software. The W-Au tips $(\phi=30-50 \mu \mathrm{m})$ were placed inside a sealed chamber. Vacuum conditions down to $\mathrm{P}=10^{-5}$ mbar were achieved. Besides, pure $\mathrm{O} 2$ atmosphere at different pressures were used in the experiments (0.074, 0.22 and 0.019 mbar). The electrical characterization of the 3-T devices was performed using either the Keithley 4200Semiconductor Characterization System or the Agilent 4156C Semiconductor Parameter Analyzer in a shielded Faraday box. I-V measurements were conducted through two probes which have been configured by software to apply voltage ramps while sensing the current. The device has been biased by locating the measuring probes on top of the $\mathrm{G}$ and $\mathrm{C}$ electrodes (Figure 3d) used for writing/erasing the $\mathrm{CeO}_{2-\mathrm{x}} / \mathrm{LSMO}$ bridge under the gate electrode area. Positive voltage was applied to the $\mathrm{C}$ electrode while the $\mathrm{G}$ electrode was electrically grounded. In the case of the LSMO/STO films, the tips were placed in direct contact with the bare LSMO surface

Materials characterization: The microstructure of the films was revealed by Transmission Electron Microscopy (TEM) and Scanning Transmission Electron Microscopy (STEM) techniques. Different equipment was used in the experiments: an FEI Tecnai G2 F20 operated at $200 \mathrm{kV}$, an FEI Titan 60-300, microscope equipped with an X-FEG gun, a CETCOR probe corrector and a Gatan TRIDIEM 866 ERS energy filter, operated in STEM mode at $300 \mathrm{kV}$. The samples were thinned by mechanical grinding, polishing, and dimpling, followed by Arion milling. The resistivity measurements as a function of temperature were performed in a Physical Properties Measurement System (PPMS) using a 4-point configuration.

\section{Supporting Information}

Supporting Information is available from the Wiley Online Library or from the author.

\section{Acknowledgements}


We acknowledge financial support from Spanish Ministry of Economy and Competitiveness through the "Severo Ochoa” Programme for Centres of Excellence in R\&D (SEV-2015-0496), CONSOLIDER Excellence Network (MAT2015-68994-REDC), COACHSUPENERGY project (MAT2014-56063-C2-1- R, co-financed by the European Regional Development Fund), OXSWITCH project funded by Centro Superior de Investigaciones Científicas (CSIC) and from the Catalan Government with 2017-SGR-1519. We also acknowledge Dr. Jaume Gazquez and Mr. Bernat Mundet for the TEM images. J.C.G.R. thanks Spanish Ministry of Economy for his FPI Spanish grant (BES-2012-053814) and the COST action MP-1308 “TOBE” (Grant COST-STSM-MP1308-070316-071463) from the European Union. R.O. thanks Generalitat de Catalunya for the Grant through AGAUR FI-DGR program. JS acknowledges the support of the Generalitat de Catalunya under the ICREA ACADEMIA award. T.P., A.P., J.S. and X.O. conceived the experiments. J.C.G.-R., R.O.-H. and M.C. growth the samples and microfabricated the devices. T.P., R.D., B.A. and J.C.G.R. designed the atmospheredependent measurements. J.C.G.R and B.A. performed those experiments. R.O-H. performed the 3-T device experiments. J.C.G.R., T.P., A.P., X.O., R.O.-H. wrote the manuscript. All authors contributed to the discussion and implications on the at all stages.

Received: ((will be filled in by the editorial staff))

Revised: ((will be filled in by the editorial staff)) Published online: ((will be filled in by the editorial staff))

\section{References}

[1] L. O. Chua, IEEE Trans Circuit Theory, 1971, CT18, 507.

[2] D. B. Strukov, G. S. Snider, D. R. Stewart, R.S. Williams, Nature, 2008, 453, 8.

[3] Z. Wang, S. Joshi1, S. E. Savel'ev, H. Jiang, R. Midya, P. Lin, M. Hu, N. Ge, J. P. Strachan, Z. Li, Q. Wu, M. Barnell, G.-L. Li, H. L. Xin, R. S. Williams, Q. Xia, J. J. Yang, Nat. Mater. 2016, 16, 101.

[4] D. S. Jeong, R. Thomas, R. S. Katiyar, J. F. Scott, H. Kohlstedt, A. Petraru, C. S. Hwang, Reports Prog. Phys. 2012, 75, 076502.

[5] Y. V. Pershin, M. Di Ventra, Adv. Phys. 2011, 60, 145-227.

[6] R. Waser, R. Dittmann, G. Staikov, K. Szot, Adv. Mater. 2009, 21, 2632.

[7] J. J. Yang, D. B. Strukov, D. R. Stewart, Nat. Nanotechnol. 2013, 8, 13.

[8] Z. K. Yang, S. Ramanathan, Annu. Rev. Mater. Res. 2011, 41, 337.

[9] E. Janod, J. Tranchant, B. Corraze, M. Querré, P. Stoliar, M. Rozenberg, T. Cren, D. 
Roditchev, V. T. Phuoc, M.-P. Besland, L. Cario, Adv. Funct. Mater. 2015, 25, 6287.

[10] J. C. Gonzalez-Rosillo, R. Ortega-Hernandez, J. Jareño-Cerulla, E. Miranda, J. Suñe, X. Granados,X. Obradors, A. Palau, T. Puig, J. Electroceramics 2017, 39, 185.

[11] T. L. Meyer, R. Jacobs, D. Lee, L. Jiang, J. W. Freeland, C. Sohn, T. Egami1, D. Morgan, H. N. Lee. Nat. Commun. 2018, 9, 1.

[12] C. Moreno, C. Munuera, S. Valencia, F. Kronast, X. Obradors, C. Ocal, Nano Lett. 2010, 10, 3828.

[13] R. Ortega-Hernandez, M. Coll, J. Gonzalez-Rosillo, A. Palau, X. Obradors, E. Miranda, T. Puig, J. Suñe, Microelectron. Eng. 2015, 147, 37.

[14] Y. Zhou, S. Ramanathan, Crit. Rev. Solid State Mater. Sci. 2013, 38, 286.

[15] J. Jeong, N. Aetukuri, T. Graf, T. D. Schladt, M. G. Samant, S. S. P. Parkin, Science. 2013, 339, 1402.

[16] N. Banno, T. Sakamoto, T. Hasegawa, K. Terabe, M. Aono, Japanese J. Appl. Physics, 2006, 45, 3666.

[17] A. J. Grutter, D. A. Gilbert, U. S. Alaan, E. Arenholz, B. B. Maranville, J. A. Borchers, Y. Suzuki, Kai Liu, B. J. Kirby. Appl. Phys. Lett. 2016, 108, 082405.

[18] L. Yao, S. Inkinen, S. van Dijken, Nat. Commun. 2017, 8, 14544.

[19] Y. van de Burgt, E. Lubberman, E. J. Fuller, S. T. Keene1, G. C. Faria, S. Agarwal, M. J. Marinella5, A. A. Talin, A. Salleo, Nat. Mater. 2017, 16, 414

[20] Pickett, M. D., Medeiros-Ribeiro, G. \& Williams, R. S. A scalable neuristor built with Mott memristors. Nat. Mater. 2012, 12, 114-117.

[21] Shi, J., Ha, S. D., Zhou, Y., Schoofs, F. \& Ramanathan, S. A correlated nickelate synaptic transistor. Nat. Commun. 2013, 4, 2676.

[22] Ha, S. D. \& Ramanathan, S. Adaptive oxide electronics: A review. J. Appl. Phys. 2011, $110,071101$.

[23] F. Messerschmitt, M. Kubicek, J. L. M. Rupp, Adv. Funct. Mater. 2015, 25, 5117.

[24] Z. A. Feng, F. El Gabaly, X. Ye, Z.-X. Shen, W. C. Chueh, Nat. Commun. 2014, 5, 4374.

[25] D. Cooper, C. Baeumer, N. Bernier, A. Marchewka, C. La Torre, R. E. DuninBorkowski, S. Menzel, R. Waser, R. Dittmann, Adv. Mater. 2017, 29, 1700212.

[26] P. Gao, Z. Wang, W. Fu, Z. Liao, K. Liu, W. Wang, X. Bai, E. Wang, Micron 2010, 41, 301.

[27] S. Menzel, U. Böttger, M. Wimmer, M. Salinga, Adv. Funct. Mater. 2015, 25, 6306.

[28] R. Merkle, J. Maier, Angew. Chemie - Int. Ed. 2008, 47, 3874.

[29] J. Fleig, R.Merkle,J. Maier, Phys. Chem. Chem. Phys. 2007, 9, 2713.

[30] Y.A. Mastrikov, R. Merkle, E. Heifets, E. A. Kotomin, J. Maier, J. Phys. Chem. C 2010, 114, 3017.

[31] P. Cayado, C. F. Sanchez-Valdes, A. Stangl, M. Coll, T. Puig, X. Obradors Phys. Chem. Chem. Phys. 2017, 19, 14129.

[32] V. Alexandrov, S. Piskunov, Y. F. Zhukovskii, E. A. Kotomin, J. Maier, Integr. Ferroelectr. 2001, 123, 10.

[33] M. M. Kuklja, E.A. Kotomin, R. Merkle, Y. Mastrikov, J. Maier, Phys. Chem. Chem. Phys. 2013, 15, 5443.

[34] M. Coll, J. Gazquez, A. Palau, M. Varela, X. Obradors, T. Puig† Chem. Mater. 2012, 24, 3732.

[35] M. Coll, A. Palau, J.C. Gonzalez-Rosillo, J. Gazquez, X. Obradors, T. Puig Thin Solid Films 553, 7-12 (2014).

[36] A. Palau, A. Fernandez-Rodriguez, JC Gonzalez-Rosillo, X. Granados, M. Coll, B. Bozzo, R. Ortega-Hernandez, J. Suñe, N. Mestres, X. Obradors and T. Puig, ACS Appl Mater Interfaces. 2018, 10, 30522-30531

[37] X. Chen, N. Wu, J. Strozier, A. Ignatiev. Appl Phys Lett. 2006, 89, 063507. 
[38] EJ Fuller, F. El Gabaly, F. Léonard, S. Agarwal, S.J. Plimpton, R.B. Jacobs-Gedrim, C.D. James, M.Jm Marinella, A.A. Talin, Adv Mater. 2017, 29, 1-8.

[39] Q. Xia, MD Pickett, JJ. Yang, X. Li, W. Wu, G. Medeiros-Ribeiro, R.S. Williams. Adv Funct Mater. 2011, 21, 2660-2665.

[40] P.B. Pillai, M.M. de Souza, ACS Appl. Mater. Interfaces, 2017, 9(2), 1609-1618

[41] E. Herrmann, A. Rush, T. Bailey, R. Jha, IEEE Electron Device Lett. 2018, 39, 500503

[42] C. Acha, A. Schulman, M. Boudard, K. Daoudi, T. Tsuchiya, Appl. Phys. Lett. 2016, 109, 011603.

[43] Z. Li, M. Bosman, Z. Yang, P. Ren, L. Wang, L. Cao, X. Yu, C. Ke, M.B.H. Breese, A. Rusydi, W. Zhu, Z. Dong and Y.L. Foo, Adv. Funct. Mater. 2012, 22, 4312-4321

[44] H. Guo, J. Wang, X. He, Z. Yang, Q. Zhang, K. Jin, C. Ge, R. Zhao, L. Gu, Y. Feng, W. Zhou, X. Li, Q. Wan, M. He, C. Hong, Z. Guo, C. Wang, H. Lu, K. Ibrahim, S. Meng, H. Yang, G. Yang, Adv. Mater. Interfaces 2016, 3, 1500753

[45] J. Lia, C. K. Ong, J.-M. Liu, Q. Huang, S. J. Wang, Appl. Phys. Lett. 2000, 76, 8, 1051

[46] P. Orgiani, A. Yu. Petrov, R. Ciancio, A. Galdi, L. Maritato, B. A. Davidson, Appl. Phys. Lett. 2012, 100, 042404

[47] Z. L. Wang, J. S. Yin, and Y. D. Jiang, J. Zhang, Appl. Phys. Lett., 1997, 70 (25), 3362




Figure 1. Resistive Switching characteristics in different atmospheres. a) Scheme of the measurement setup in bare LSMO films with the tips in direct contact with the surface and TEM micrograph. The surface of the film is biased and the tip, under which the switching event occurs, is grounded. b) I-V sweeps of LSMO films in ambience and vacuum conditions. c) I-V sweeps at different oxygen partial pressures. d) Cumulative probability plots derived from b). e) Cumulative probability plot of the I-V sweeps derived from c). f) Scheme of the measurement setup in $\mathrm{Ag} / \mathrm{CeO}_{2-\mathrm{x}} / \mathrm{LSMO}$ cell and TEM micrograph. g) I-V sweeps of the $\mathrm{Ag} / \mathrm{CeO}_{2-\mathrm{x}} / \mathrm{LSMO}$ cells in ambience and vacuum conditions. h) Cumulative probability plot derived from g). In the cumulative plots the LRS have been indicated with spheres while the HRS have been represented with stars.

a)

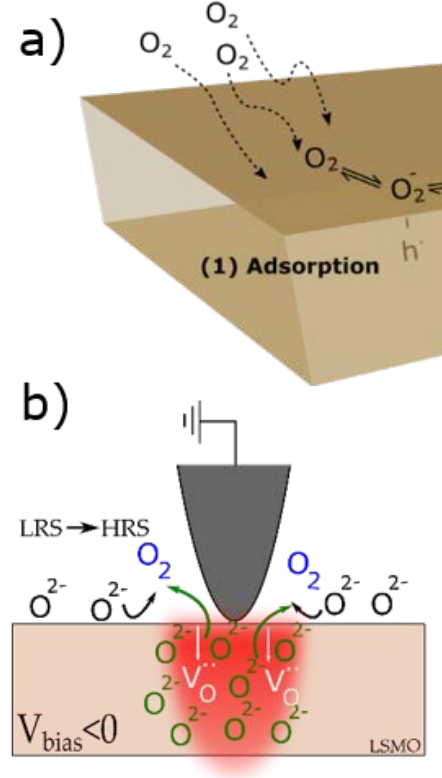

$\mathrm{O}_{2} \quad \mathrm{O}_{2}$
(3) Surface migration of adsorbates

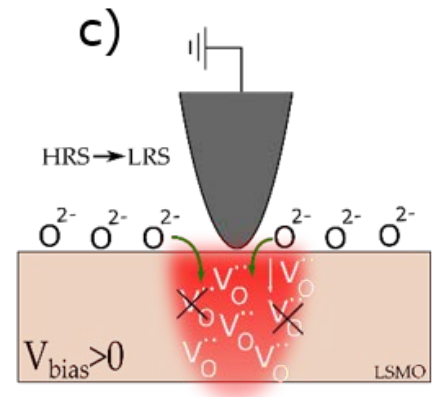

d)

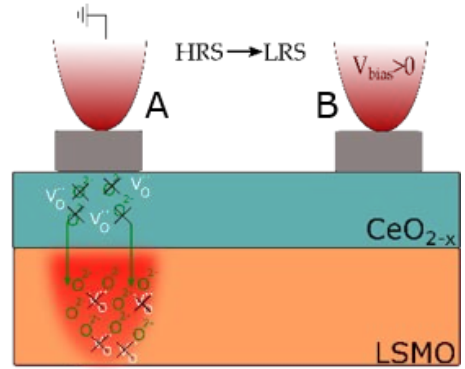

e)

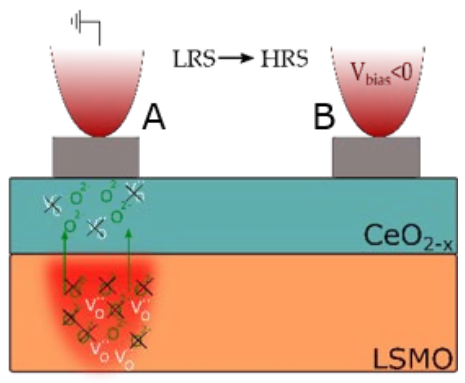

Figure 2. Oxygen exchange mechanisms. a) Sketch showing the main steps in the oxygen incorporation/release processes. The final amount of oxygen adsorbates depends on the $\mathrm{p}\left(\mathrm{O}_{2}\right)$. b) Scheme of the oxygen release process in LSMO films. $\mathrm{O}_{2}$ from the crystal electrochemically react with an adsorbate to form an $\mathrm{O} 2$ molecule and creating an oxygen vacancy in the system. c) Scheme of the incorporation step in LSMO films. Oxygen adsorbates are driven by the electric field into the crystal lattice annihilating an oxygen vacancy. d) and e) Oxygen ion exchange mechanism between the $\mathrm{La}_{0.8} \mathrm{Sr}_{0.2} \mathrm{MnO}_{3}$ and the $\mathrm{CeO}_{2-\mathrm{x}}$ layers proposed to describe the resistive switching phenomenon according to the bias polarization. 


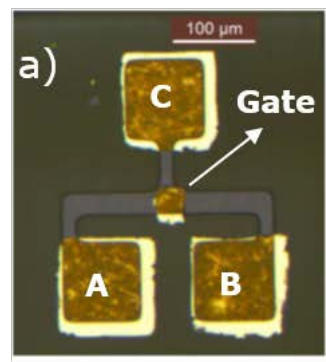

d)

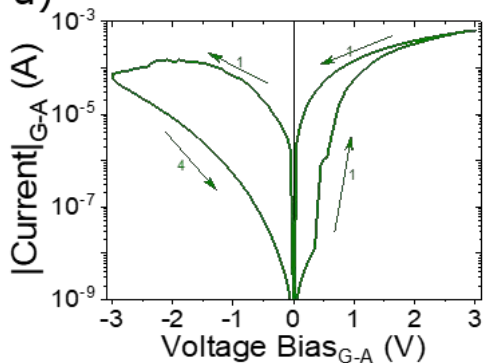

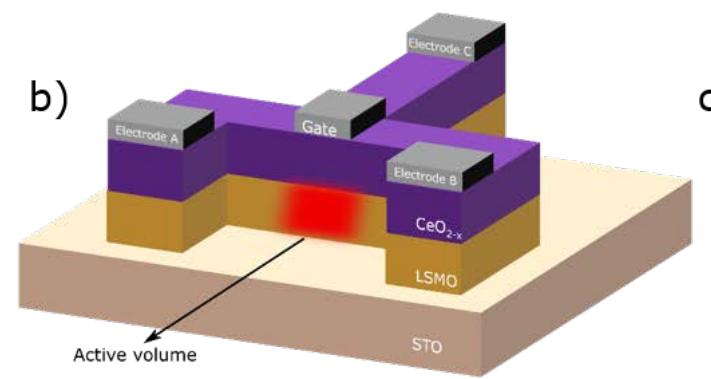

c)

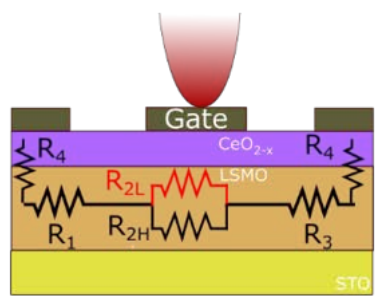

f)



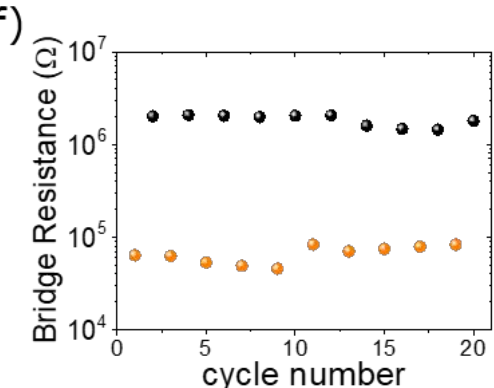

Figure 3. Proof-of-concept 3-Terminal device. a) Optical micrograph (top view) of the 3-T $\mathrm{Ag} / \mathrm{CeO}_{2-\mathrm{x}} / \mathrm{LSMO}$ device. The writing/erasing (W/E) voltage is applied between the gate $\mathrm{G}$ and the $\mathrm{C}$ electrodes while the actual state of the track is evaluated when applying voltage between the A-B electrodes. b) Scheme of the 3-T device. The red region under the Gate electrode indicates the active switching region, i.e., the full LSMO volume below the G electrode. c) Equivalent circuit representing two different resistance states of the material under the gate electrode which has been electrically modified. R2L represents the $\mathrm{La}_{0.8} \mathrm{Sr}_{0.2} \mathrm{MnO}_{3}$ in the LRS while R2H in the HRS d) Bipolar RS induced after the initialization steps in the3-T device when the measurement probes are located on top of the $G$ and the $\mathrm{C}$ electrodes. The voltage sweep direction is indicated by the arrows. e) Measured curves between $\mathrm{A}$ and $\mathrm{B}$ electrodes used to read the actual state of the bridge in a 3-T device after each writing/erasing process. The initial HRS of the track is changed to a LRS by inducing the volume resistive switching under the gate electrode. Then the HRS is recovered by switching back the region under the gate electrode. f) Resistance state measured at $0.5 \mathrm{~V}$ on the bridge with good repeatability up to at least 20 cycles and resistance ratio of $\sim 15$ 
The increasing interest in advanced nanoelectronics applications, such as neuromorphic computing and Resistive Random Access Memories, demands a deep understanding of ionic migration to engineer novel material functionalities. In this article, we demonstrate the opportunities of $\mathrm{La}_{1-\mathrm{x}} \mathrm{Sr}_{\mathrm{x}} \mathrm{MnO}_{3-\mathrm{y}}$ to develop a novel Volume Resistive Switching effect determined by the Metal-Insulator Transition characteristics of this strongly correlated system.

Keyword: Resistive Switching

Juan Carlos Gonzalez-Rosillo, Rafael Ortega-Hernandez, Benedikt. Arndt, Mariona Coll1, Regina Dittmann, Xavier Obradors, Anna Palau, Jordi Suñe and Teresa Puig

Engineering Oxygen Migration for Homogeneous Volume Resistive Switching in 3Terminal Devices

Understanding oxygen exchange...

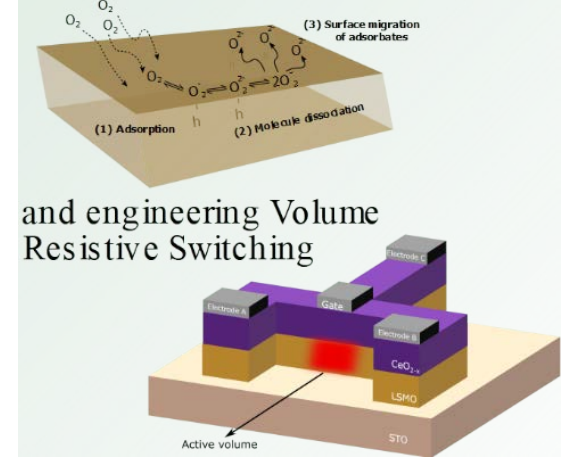




\section{Supporting Information}

\section{Engineering Oxygen Migration for Homogeneous Volume Resistive Switching in 3- Terminal Devices}

Juan Carlos Gonzalez-Rosillo, Rafael Ortega-Hernandez, Benedikt. Arndt, Mariona Coll1, Regina Dittmann, Xavier Obradors, Anna Palau, Jordi Suñe and Teresa Puig

After $\mathrm{La}_{0.8} \mathrm{Sr}_{0.2} \mathrm{MnO}_{3}$ (LSMO) thin film deposition the evaluation of their physical and structural properties was carried out in order to characterize this layer. The temperature dependence of resistivity $\rho(T)$ was measured with the 4-point configuration using a Physical Properties Measurement System (PPMS, Quantum Design). Figure S.1 shows the resistivity behavior of $\mathrm{La}_{0.8} \mathrm{Sr}_{0.2} \mathrm{MnO}_{3}$ films grown on STO substrate where the metal-insulator transition (MIT) has been observed. In the figure, the arrow indicates the MIT temperature at 310K.

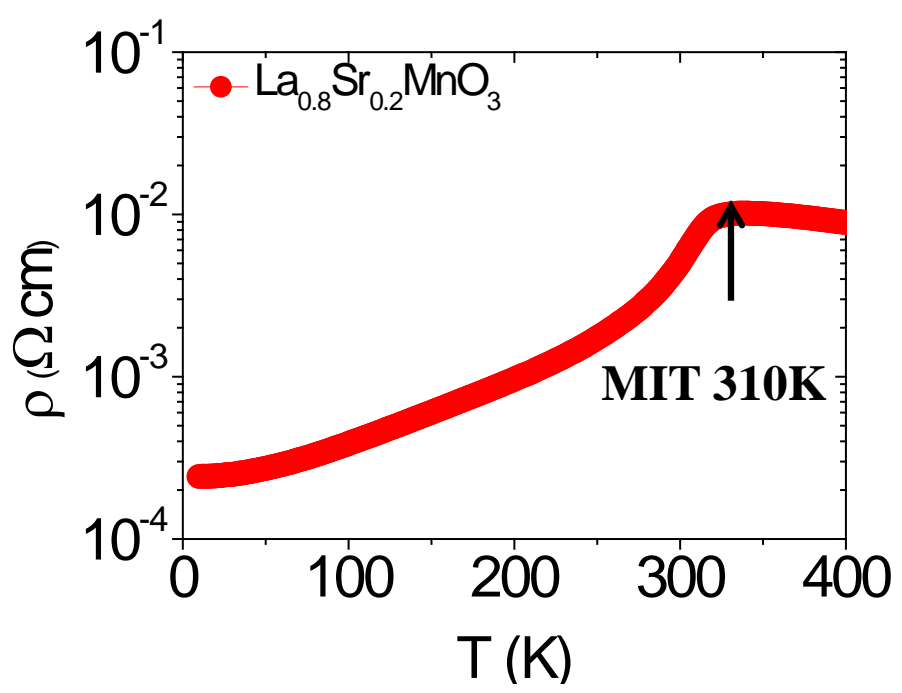

Figure S1. Resistivity measurements as a function of temperature measured in a $40 \mathrm{~nm}$ thickness $\mathrm{La}_{0.8} \mathrm{Sr}_{0.2} \mathrm{MnO}_{3}$ layer. The measured MIT temperature is $\mathrm{T}_{\mathrm{MIT}} \sim 310 \mathrm{~K}$

During the evaluation of the resistive switching characteristics in a 3-Terminal fresh device, an initialization step is necessary in order to induce the bipolar resistive switching. This event turns the device from a very high insulating state (branch 1 in figure S.2) to a less resistive one but without reaching completely the LRS (branch 2 in figure S.2) of the resistive switching phase. In figure S.2 the initialization event is presented when $-5 \mathrm{~V}$ is applied in a fresh device. At the subsequent bias, the BRS behaviour is confirmed according to the bias polarization (branches 3-6). 




Figure S2. Initialization step observed in branches 1-2 during the evaluation of a bilayer fresh device. This event turns the device from a very high insulating state to a less resistive state but without reaching the low resistance state.

Related to a three terminal device, it is worth noting that this initialization event does not modify the actual resistance state at the track region under the gate electrode. This statement is confirmed by measuring the resistance state before and after the electroforming process applying a low voltage measurement of $\pm 1 \mathrm{~V}$ between the A-B electrodes. In figure S.3 the "before initialization step” and "after initialization step” curves are superimposed one each other indicating that any resistance modification has been produced along the bilayer bridge at this stage.

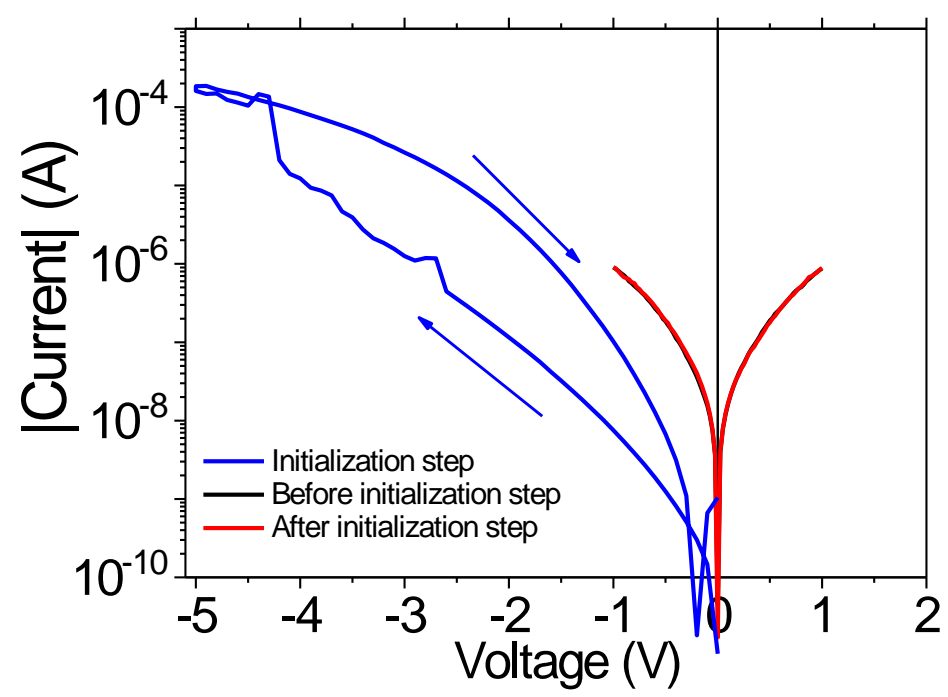

Figure S3. In this figure, one can observe that the electroforming event does not modify the resistance state along the track when evaluating a three-terminal device. The "before electroforming" and "after electroforming” curves are superimposed one each other 
indicating that any modification has been produced at this stage.

From a circuit point of view, different resistance values are observed when the resistive switching is produced under the gate electrode as shown in figure 3 in the manuscript. The resistance of the transformed area in the LRS can be calculated using the following expression modified from the resistivity equation ${ }^{1}$ :

$$
R_{2 L}=\rho_{\text {LSMO }} \frac{\mathrm{L}}{\mathrm{W}\left(\mathrm{t}_{\text {LSMO }}-\mathrm{x}\right)}
$$

The $\rho_{\text {LSMO }}$ and $t_{L S M O}$ are the resistivity and the thickness of the LSMO layer respectively. The $x$ factor indicates the part of the LSMO thickness that has been switched to the LRS. The equivalent resistance $R_{e q}$ of the device, as indicated in the figure 3c, is presented as follows:

$$
R_{e q}=\frac{R 2 L \cdot R 2 H}{R 2 L+R 2 H}
$$

From our resistance ratio measurements in the bipolar resistive switching of $\sim 10^{3}$, we can make an approximation that the value of the $R 2 B$ is $R 2 H=10^{3} R 2 L$. Substituting this value in eq. 2 we obtain:

$$
\begin{gathered}
R_{e q}=\frac{R 2 L \cdot 10^{3} R 2 L}{R 2 L+10^{3} R 2 L} \\
R_{e q}=\frac{R 2 L \cdot 10^{3} R 2 L}{R 2 L\left(1+10^{3}\right)} \\
R_{e q}=R 2 L=\rho_{L S M O} \frac{L}{W\left(t_{L S M O}-x\right)}
\end{gathered}
$$

The part of the transformed thickness can be known provided that all the parameters related to the geometry of the transformed area and resistivity are known. However, we only know that the resistance ratio measured along the track between the LRS and the HRS is around 15. The expression relating these parameters is presented as follows: 


$$
R_{\text {ratio }}=\frac{R_{\text {HRS }}}{R_{\text {LRS }}}=\frac{R 1+R 2+R 3}{R 1+R_{\text {eq }}+R 3}
$$

The $\mathrm{R}_{\mathrm{HRS}}$ and $\mathrm{R}_{\mathrm{LRS}}$ of the track have been already measured when the cycles were evaluated. In addition, the resistances $R 1$ and $R 3$ can be easily calculated due to the fact that device geometry is known.R4 has not been included since it would only add a constant term. Further experiments are required to elucidate the exact contribution of the $\mathrm{CeO}_{2}$ to the device resistance. From the design of the three terminal structures we know the dimensions of the devices and the resistivity of the LSMO in the LRS. The values of $L=140 \mu \mathrm{m}$ and $W=30 \mu \mathrm{m}$, with the LSMO resistivity of $4.8 \mathrm{~m} \Omega$-cm with thickness of $40 \mathrm{~nm}$ results in a resistance value of $5.6 \mathrm{k} \Omega$. In addition, due to the fact that the low voltage measurements do not produce any kind of resistive switching in other parts on the track just under the gate electrode, we can assure that due to the symmetry of the device the $R 1$ and $R 3$ remain both with the same resistance value, i.e., $R 1=R 3$. As a result, the $R_{L R S}$ can be rewritten as:

$$
R_{\text {LRS }}=2 R 1+R_{\text {eq }}
$$

Substituting the values of $R_{L R S}, R_{H R S}$ and $R_{\text {ratio }}$ in equation 4.6 we can obtain the value of $R_{e q}$ as follows:

$$
\begin{gathered}
R_{\text {ratio }}=\frac{R_{\text {HRS }}}{2 R 1+R_{\text {eq }}} \\
15=\frac{1 \times 10^{6} \Omega}{2\left(5.6 \times 10^{3} \Omega\right)+R_{\text {eq }}} \\
R_{\text {eq }}=55.4 \mathrm{k} \Omega
\end{gathered}
$$

The resistance of the transformed area is now known. Rewriting equation 5 is possible to know the thickness of the LSMO layer that has been switched using the length $(40 \mu \mathrm{m})$ and the width $(30 \mu \mathrm{m})$ of the gate electrode.

$$
\begin{gathered}
x=t_{\text {LSMO }}-\rho_{\text {LSMo }} \frac{L}{R_{e q} \cdot W} \\
x=40 \times 10^{-9} m-4.8 \times 10^{-5} \Omega m \frac{40 \times 10^{-6} m}{55.4 \times 10^{3} \Omega \cdot 30 \times 10^{-6} m}
\end{gathered}
$$




$$
x=38.8 \mathrm{~nm}
$$

This value reveals that the volume switching achieved in the $\mathrm{La}_{0.8} \mathrm{Sr}_{0.2} \mathrm{MnO}_{3}$ layer transforms its whole thickness which is in agreement with our observations reported before ${ }^{2}$.

Small area dependence of Ag/CeO2-x/LSMO devices, further supporting the area dependent volumetric switching event localization. The complex area dependent of the system will be further addressed in an extended range in future publications.



Figure S4. Area dependence of the switching in $\mathrm{Ag} / \mathrm{CeO} 2 / \mathrm{LSMO}$ devices

Preliminary pulsing data have revealed switching times in the ns (100 ns for pulses $\sim 4 \mathrm{~V}$ ). The final resistive state can be tuned by controlling the voltage-time parameters of the pulse.

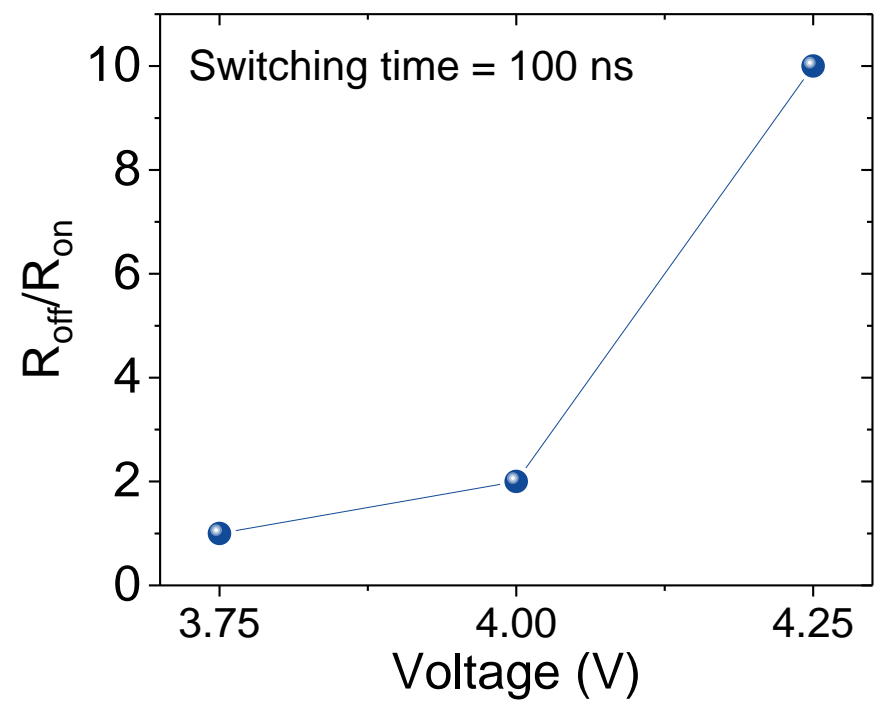


Figure S5. Area dependence of the switching in Ag/CeO2/LSMO devices
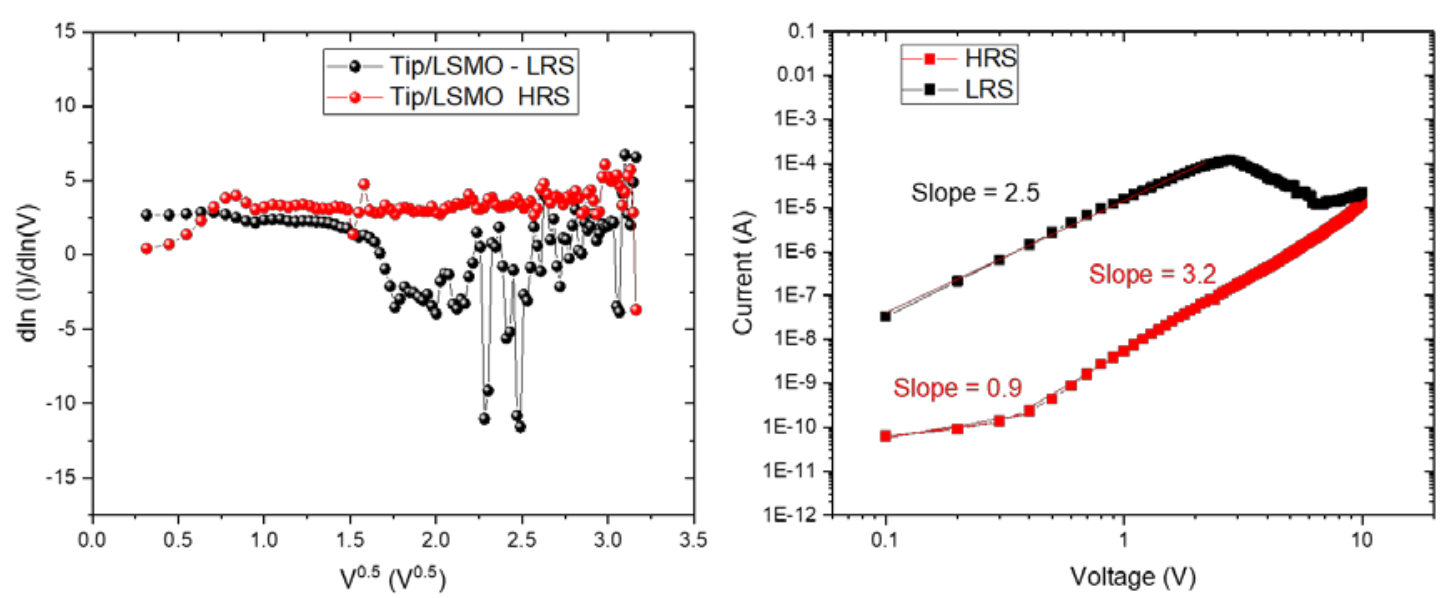

Figure S6. $d(\operatorname{Ln}(\mathrm{I})) / \mathrm{d}(\operatorname{Ln}(\mathrm{V}))$ vs V0.5 and Log I-Log V profiles for the tip/LSMO

Electrical readout by C-AFM of the region where a switching event was induced with a fresh probe on a bare LSMO film. It can be shown that the region switched presents around 10-15 $\mu \mathrm{m}$ in diameter.

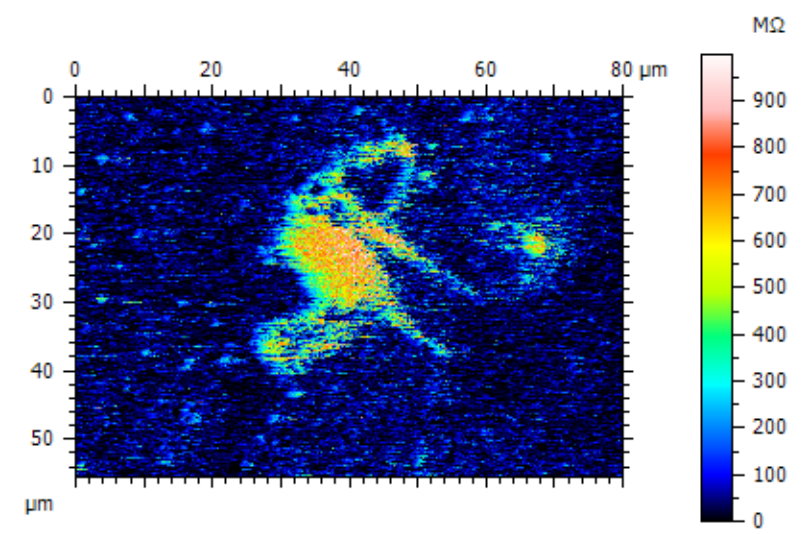

1. Schroder, D. K. Semiconductor Material and Device Characterization. (John Wiley \& Sons, 2006).

2. Gonzalez-Rosillo, J. C. et al. Volume Resistive Switching in metallic perovskite oxides driven by the Metal-Insulator Transition. J. Electroceramics 1-12 (2017). doi:10.1007/s10832-017-0101-2 